\title{
Spatial coding of word-initial letters: Evidence from a Simon-like task
}

\author{
BARBARA TRECCANI \\ University of Padua, Padua, Italy \\ and University of Edinburgh, Edinburgh, Scotland \\ Roberto Cubelli \\ University of Trento, Trento, Italy \\ Sergio Della Sala \\ University of Edinburgh, Edinburgh, Scotland \\ AND \\ CARLo Umiltà \\ University of Padua, Padua, Italy
}

\begin{abstract}
In this study, we investigated whether the processing of written words leads to a preferential coding of word beginnings and whether this coding occurs in the context of word representations that are spatial in nature and depend on the orientation of the actual stimuli. Two experiments were carried out wherein participants were asked to press a left or right key, in accordance with a nonspatial feature of standard-oriented or mirror-reversed wordlike stimuli (words and pronounceable nonwords). Both experiments showed an effect of correspondence between position of the beginning part of the stimuli and position of the required response (i.e., a Simon-like effect): Responses to standard-oriented stimuli were faster with the left key, whereas responses to mirror-reversed stimuli were faster with the right key. The present findings indicate for the first time that, in reading, the direction of script is automatically processed and the position of the word beginning is coded before the orthographic lexicon is accessed.
\end{abstract}

People can read words regardless of the orientation in which they are written. Readers can access a word starting from any orientation of the physical stimulus-for example, standard, which in Western orthographies is horizontal and from left to right, or mirror reversed, from right to left. This requires the recognition of the direction of the script, which can be achieved on the basis of the letter structure and shape (see Asso \& Wyke, 1967) and the conversion of the spatial information (e.g., left-right), relative to the different parts of the word stimulus, into ordinal information (beginning-end).

This is consistent with the model of word recognition proposed by Caramazza and Hillis (1990), according to which prelexical word representations are spatial in nature. Starting from a stimulus-centered description that adheres to the physical stimulus features, a canonical word-centered representation is computed. The former describes the shape and the local position of each letter form relative to other letter forms in the stimulus (e.g., the initial letters of standard-oriented and mirror-reversed words as the leftmost and rightmost letter forms, respectively), whereas the latter is an abstract orthographic representation that specifies the identity and order of the letters in terms of their relative po- sitions in the word, independently of the orientation of the physical stimulus (e.g., the initial letter of either a standardoriented or a mirror-reversed word as the leftmost letter).

Specific features of prelexical representations are still debated. A controversial topic concerns whether or not the availability and quality of represented information differs as a function of letter position, which, in turn, is linked to the way these representations are held to be computed. Words are generally thought to be processed as single items, without a letter-by-letter attentional scan, and to produce holistic, internally uniform representations, at least where reading is concerned. The seminal study by LaBerge (1983), for example, showed that when participants had to categorize a word, reaction times (RTs) to probe stimuli subsequently presented in various parts of the word did not differ from one another. In contrast, when participants had to categorize a single letter of the word, RTs increased as a function of the probe-letter distance. This would suggest that, when words are read, attention is equally distributed across the component letters; that is, the attention "spotlight" covers the whole word (Inhoff, Pollatsek, Posner, \& Rayner, 1989; see also Sieroff \& Posner, 1988). This is consistent with the widely held belief

B. Treccani, barbara.treccani@unipd.it 
that the letters of a word are processed in parallel (e.g., Plaut, McClelland, Seidenberg, \& Patterson, 1996).

In spite of this idea's being shared by the majority of word recognition theories, it has been proposed that different parts of a word are not equally represented but that prelexical representations are biased toward word beginnings. According to some authors, this is the consequence of attentional focusing on word-initial letters to establish a cohort of lexical entries, among which the correct one is then individuated. Attention could be selectively oriented toward these letters either because they contain more useful information for recognition (i.e., they include more of the linguistic information that distinguishes a given word from other words, thus limiting the number of potential word interpretations; see, e.g., Brysbaert, Vitu, \& Schroyens, 1996; Farid \& Grainger, 1996) or simply because lexical access starts from them (Kwantes \& Mewhort, 1999) independently of their information value (Fischer, 2000). According to another view (Montant, Nazir, \& Poncet, 1998), given that, in reading, the eyes tend to land to the left of the word's center (i.e., the so-called preferred landing position [PLP] effect; Rayner, 1979), the reading system is trained to perceive word-initial letters with higher resolution than final letters. As a consequence, it "learns" to attribute a higher weight to initial letters' information, which would then be better represented. Finally, a wordbeginning bias in prelexical representations might result directly from the way in which letter position is encoded. According to Whitney (2001), the position of letters in a word is coded in terms of their relative activation level: There is an activation gradient that decreases monotonically across letter positions, with the highest value for the initial letter and the lowest value for the final letter.

Evidence in support of a word-beginning bias in prelexical processing is highly controversial. Studies that have addressed the issue of the relative importance of specific letter positions in visual word recognition have yielded inconsistent results. Montant et al. (1998) showed that performance in a lexical decision task was more accurate for nonwords that differed from real words in their initial letters, rather than their medial or final letters. Yet the results obtained with other paradigms (e.g., orthographic priming induced by nonwords) have failed to demonstrate enhanced processing of word beginnings. For example, Johnson, Perea, and Rayner (2007) showed that nonwords created by transposing internal letters of real words are perceived as more similar to their base words than are replaced-letter nonwords (e.g., jugde is more easily perceived as judge than is junpe). This effect is smaller, or even absent, for both initial and final letters, indicating that exterior letters may be critical in visual word recognition, but there is no evidence suggesting a more important role for initial than for final letters (see also Schoonbaert \& Grainger, 2004).

Word-beginning biases have also been investigated by testing possible asymmetries in the position at which people accomplish certain operations within words. The results from studies that have examined this question have not been clear-cut either. Fischer $(1996,2000)$ showed that when participants are asked to make a bisection mark at the center of horizontally printed words and pronounce- able nonwords, they tend to bisect these stimuli to the left of the actual midpoint. According to Fischer, the bisection bias is the consequence of orienting attention toward the word beginning, which would lead to an overrepresentation of its physical extent, and is linked to the PLP effect (i.e., the attention movement is followed by a corresponding eye movement). Both the bias in word bisection and the PLP effect might result from high-level strategies aimed at landing attention and eyes on the word beginning, where readers know useful information is likely to be found (see also, e.g., Farid \& Grainger, 1996). However, the high-level accounts of the word bisection bias and PLP effect have received little support (for a discussion, see, e.g., Ducrot \& Pynte, 2002; Vonk, Radach, \& van Rijn, 2000). Most studies suggest that both phenomena are due to low-level factors that are not specific to reading. The PLP effect has usually been observed in continuous reading and may result from oculomotor constraints associated with saccade execution through stimuli arranged in sequence (i.e., words in the text lines) according to the reading direction (e.g., McConkie, Kerr, Reddix, \& Zola, 1988): The eyes are not oriented toward the word beginning but toward the word center, and they land to the left (in left-to-right readers), owing to the properties of the oculomotor system (i.e., saccades would undershoot their goal). Indeed, when the eyes are already fixating the word center, the probability of refixating the word (either on the left or on the right) is very small (e.g., Vitu, O'Regan, \& Mittau, 1990). Recently, Ducrot and Pynte showed PLP effects and systematic biases in a bisection task of isolated words, which were presented on the left or on the right of the initial fixation point. These effects, however, were also observed with other stimuli composed of discrete elements (e.g., strings of hashes) and appeared to be related to the participants' reading habits. These results are consistent with those of Chokron and Imbert (1993), who showed leftward and rightward biases in a line bisection task performed by left-to-right and right-toleft readers, respectively. These findings can be accounted for by assuming that there is a tendency to scan stimuli according to reading direction. This tendency is stronger when discrete, rather than continuous, stimuli are used and causes both the PLP effect (e.g., English readers land left of stimulus center so as to be able to scan the stimulus from left to right) and the bisection bias (i.e., when scanning the stimulus in a bisection task, participants stop short of the true midpoint - e.g., on the left if the scanning direction is from left to right).

This explanation is reminiscent of that proposed to explain left-right asymmetries in the processing of elements of discrete stimuli observed with selective attention tasks. For example, in flanker tasks (Eriksen \& Eriksen, 1974), wherein participants are asked to discriminate the central letter of a string, letters to the left of the target cause a greater interference effect (i.e., longer RTs) when they are not congruent with the target letter (e.g., KKSSS) than do right-side flanker letters (e.g., SSSKK). This left-side bias tends to reverse into a stronger effect of right-side flankers' congruency with strings composed of mirror-reversed letters (Hommel, 1995, 2003). To account for this, Hommel proposed that processing alphabetic material induces a 
tendency to scan the string from the leftmost letter toward the right or from the rightmost letter toward the left, depending on the letters' orientation, until the central target is reached.

In sum, studies to date have not provided clear evidence that the initial letter(s) of words are coded and specified as privileged features in prelexical representation: Those examining the role of specific letter positions in visual word recognition have produced inconsistent results, whereas those showing positional biases (PLP and word bisection biases) can be explained by assuming low-level factors involved in word skipping during continuous reading or in performing serial analyses to reach a certain element within a string (e.g., the stimulus midpoint in bisection tasks).

The aim of the present study was to reexamine this issue by using a task that involved word recognition (thus, not requiring searching for a certain position/element within the stimulus) and that was nevertheless sensitive to the preferential coding of a specific part of the stimulus (i.e., the word beginning). Furthermore, consistent with the idea that prelexical representations are spatial in nature (e.g., Caramazza \& Hillis, 1990), we investigated whether this coding too is spatial.

To explore this hypothesis, we capitalized on a wellknown stimulus-response compatibility phenomenon, the Simon effect (Simon \& Small, 1969), which is thought to be diagnostic of the spatial nature of representations (e.g., Umiltà \& Nicoletti, 1992). In a typical Simon task, a target stimulus is presented on the left or the right of the fixation point, and participants have to press a left or right key according to a nonspatial stimulus attribute (e.g., the stimulus color). The Simon effect refers to the fact that responses are faster, and often more accurate, when the stimulus and the response positions correspond (e.g., left-stimulus-leftresponse) than when they do not (e.g., left-stimulus-rightresponse). Most researchers agree that this effect results from the automatic coding of stimulus position, which, in turn, automatically activates the spatially corresponding response. This response would compete at the response selection stage with that selected on the basis of the taskrelevant attribute (e.g., De Jong, Liang, \& Lauber, 1994).

If the processing of words with different orientations generates different spatial codes for the word beginning's positions, responses automatically activated by these codes should interfere with correct response selection in a Simon-like task: Faster and/or more accurate responses should be observed when this code corresponds to the position of the required response (i.e., standard-oriented-words-left responses; mirror-reversedwords-right responses) than when they do not correspond (i.e., standard-oriented-stimuli-right-responses; mirrorreversed-stimuli-left-responses). In contrast, if word processing does not involve the coding of a specific part of the stimulus, or this coding occurs but is not spatial in nature, no correspondence effects should emerge.

\section{EXPERIMENT 1}

Experiment 1 was a two-choice RT task in which participants were asked to press a left- or right-side key to in- dicate whether standard-oriented or mirror-reversed letter strings were words or nonwords. Stimuli were presented at the fixation point, to prevent possible spatial effects produced by either the stimulus location or the eyes' landing on particular positions within the stimulus.

\section{Method}

Participants. Thirty-two participants (18-50 years of age), native English speakers, were recruited from the University of Edinburgh undergraduate and postgraduate population.

Stimuli. Forty-eight words were selected from the MRC Psycholinguistic Database (Coltheart, 1981). The words were three, four, five, or six letters long (12 stimuli for each word length) and contained either no symmetric letters or just one symmetric vowel (i.e., letters such as A, for which, by mirror-reversing these letters, one obtains the same shape). The initial and last letters were always asymmetric. These words were controlled for a number of linguistic variables, such as word frequency, concreteness, familiarity, and imageability. Forty-eight legal and pronounceable nonwords were created by replacing one letter of the selected words with either a symmetric or an asymmetric vowel/consonant, in accordance with the replaced letter (e.g., ber from bed). There were 192 (randomly mixed) trials, in which all 96 stimuli were presented twice, once in standard orientation (standard letters running left to right) and once in mirror-reversed orientation (mirrored letters running right to left; see Figure 1). The stimuli were shown at the center of the screen in capital letters, Arial normal font (size: 26 points). A central $0.8^{\circ} \times 0.8^{\circ}$ cross served as the fixation mark. Both the letter strings and the fixation mark were white and were presented on a black background.

Apparatus and Procedure. The participants were seated about $70 \mathrm{~cm}$ from a 14-in. color monitor screen. Trials began with presentation of a fixation cross that remained visible for $1,500 \mathrm{msec}$. An $800-\mathrm{Hz}$ acoustic warning tone was delivered at the onset of fixation and lasted $300 \mathrm{msec}$. At fixation offset, the letter string appeared and remained on the screen until a response was made, but not longer than $2,000 \mathrm{msec}$. The string offset was followed by a blank interval of $400 \mathrm{msec}$. Responses with the wrong key and with latencies in excess of 2,000 msec were counted as errors. In both cases, an auditory error feedback was given during the blank. The trials were presented in randomized order. The participants were instructed to respond by pressing one of two keys on the computer keyboard (the "d" and "l" characters), depending on the stimulus lexical status. Half of the participants were instructed to press the right key in response to a word and the left key in response to a nonword, whereas the opposite mapping was assigned to the other participants.

\section{Results and Discussion}

Once RTs for erroneous responses had been excluded, a cut-off point equal to two standard deviations from each participant's RT mean was established, and outlying data
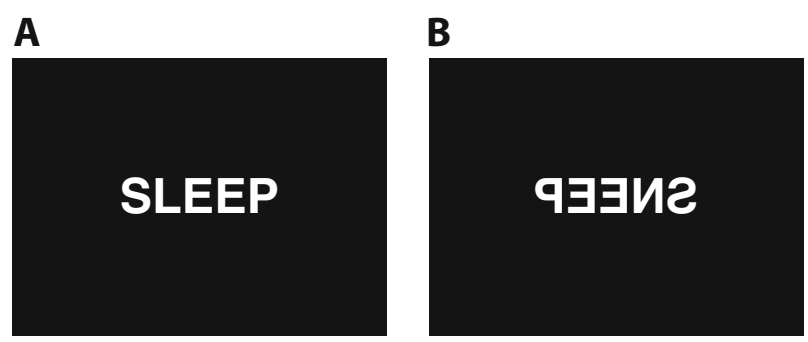

Figure 1. Experiment 1: Schematic representations of two examples of standard-oriented (A) and mirror-reversed (B) stimuli. See the text for further details. 
were replaced with that value (see Cubelli, Lotto, Paolieri, Girelli, \& Job, 2005). ANOVAs were carried out on both correct RTs and errors.

Mean correct RTs and percentages of errors are presented in Table 1. RT data and errors were submitted to ANOVAs with three within-participants factors: stimulus type (word or nonword), stimulus length (three, four, five, or six letters), and correspondence between the position of the stimulus' initial letter and the response position (corresponding or noncorresponding).

The RT ANOVA revealed significant main effects of stimulus type $[F(1,31)=82.29, p<.001]$ and stimulus length $[F(3,93)=66.30, p<.001]$ and a significant interaction between them $[F(3,93)=6.98, p<.001]$. RTs for words were shorter than those for nonwords (693 vs. $873 \mathrm{msec}$; i.e., the lexicality effect) and became longer as stimulus length increased. RTs for three-, four-, five-, and six-letter words were 714, 760, 804, and $853 \mathrm{msec}$, respectively. As is shown in Table 1, the length effect was greater for nonwords than for words. A similar result was obtained in a previous study of lexical decision for standard and rotated stimuli (Koriat \& Norman, 1985). In that study, the lexical status $\times$ length interaction was modulated by stimulus orientation. A planned comparison confirmed that this was true in our data as well $[F(3,93)=$ $4.93, p<.005]$ : For both words and nonwords, the length effect increased from the standard to the mirror-reversed orientations, and this increase was larger for word stimuli (standard-oriented words did not even show a length effect; all $p \mathrm{~s}>.05$ in Newman-Keuls post hoc tests; see Frederiksen \& Kroll, 1976).

A significant effect of correspondence on RTs was found $[F(1,31)=4.42, p<.05]$ : Corresponding trials yielded shorter RTs than did noncorresponding trials (773 vs. $792 \mathrm{msec}$ ). Responses to standard-oriented stimuli were faster when they were performed with the left hand $(676 \mathrm{msec})$ than when performed with the right hand (697 msec). The opposite was true of mirror-reversed stim-

Table 1

Experiment 1 (Lexical Decision): Mean Reaction Times (RTs, in Milliseconds) and Percentages of Errors (PEs) As a Function of Stimulus Type, Stimulus Length, and Initial-Letter-Response Spatial Correspondence

\begin{tabular}{|c|c|c|c|c|}
\hline Type & $\begin{array}{c}\text { Length } \\
\text { (in Letters) }\end{array}$ & Correspondence & RT & PE \\
\hline \multirow[t]{8}{*}{ Nonword } & \multirow[t]{2}{*}{3} & Corresponding & 779 & 4.2 \\
\hline & & Noncorresponding & 791 & 7.0 \\
\hline & \multirow[t]{2}{*}{4} & Corresponding & 840 & 6.0 \\
\hline & & Noncorresponding & 876 & 10.2 \\
\hline & \multirow[t]{2}{*}{5} & Corresponding & 892 & 6.3 \\
\hline & & Noncorresponding & 921 & 6.8 \\
\hline & \multirow[t]{2}{*}{6} & Corresponding & 919 & 11.7 \\
\hline & & Noncorresponding & 963 & 10.9 \\
\hline \multirow{8}{*}{ Word } & \multirow[t]{2}{*}{3} & Corresponding & 642 & 4.9 \\
\hline & & Noncorresponding & 644 & 7.8 \\
\hline & \multirow[t]{2}{*}{4} & Corresponding & 649 & 1.0 \\
\hline & & Noncorresponding & 676 & 4.9 \\
\hline & \multirow[t]{2}{*}{5} & Corresponding & 700 & 2.9 \\
\hline & & Noncorresponding & 702 & 6.0 \\
\hline & \multirow[t]{2}{*}{6} & Corresponding & 765 & 4.7 \\
\hline & & Noncorresponding & 763 & 6.3 \\
\hline
\end{tabular}

uli (left- and right-response RTs were 888 and $865 \mathrm{msec}$ ). No other source of variance was significant.

The results of the ANOVA performed on errors mirrored those of the RT ANOVA. There was a significant effect of stimulus type $[F(1,31)=13.55, p<.001]$. Error rates were lower for words than for nonwords $(4.8 \%$ vs. $7.9 \%)$. Stimulus length was also significant $[F(3,93)=$ $4.37, p<.01]$ and interacted significantly with stimulus type $[F(3,93)=5.40, p<.005]$. The only other significant source of variance was the correspondence effect: A lower error rate for corresponding than for noncorresponding trials (5.2\% vs. $7.2 \%$ ) was observed. Responses to standard-oriented stimuli were more accurate when they were performed with the left hand than when performed with the right hand, whereas responses to mirror-reversed stimuli showed the reverse pattern (left- vs. right-response error percentages for standard and mirrored stimuli were $2.5 \%$ vs. $5.1 \%$ and $9.8 \%$ vs. $7.9 \%$, respectively).

Both the RT and the accuracy results showed an advantage for trials on which the position of the beginning part of the stimulus corresponded to the response position. The correspondence effect was not modulated by the lexical status of the stimulus and was present for both words and nonwords. These results suggest that the processing of wordlike stimuli produces a spatial code, which corresponds to the position of initial letter(s) and can interfere with response selection.

\section{EXPERIMENT 2}

It has been proposed that biases toward word beginnings might be enhanced when the task requires the processing of nonwords (Fischer, 2000; Montant et al., 1998). Accordingly, the results obtained in Experiment 1 might be specific to the lexical decision task. To test whether the correspondence effect depends on the use of specific experimental conditions or is inherent in word recognition, we administered a task in Experiment 2 that employed only words and required semantic processing, a category discrimination task.

\section{Method}

Participants. Forty-two undergraduate and postgraduate native English speakers (18-37 years of age) from the University of Edinburgh participated. None of them had taken part in Experiment 1.

Stimuli, Apparatus, and Procedure. Forty-eight words referring to the animal category and 48 words referring to the vegetable category were selected from the British National Corpus (BNC) database (Burnage \& Dunlop, 1993). The words were four, five, six, or seven letters long (12 stimuli for each word length). At least 33\% of the letters composing the words were asymmetric. The initial and last letters were always asymmetric. The two categories of words did not differ for frequency, but the mean frequency of four-letter words was significantly higher than those of longer words. All the remaining aspects of the experimental material, apparatus, and procedure were the same as those in Experiment 1, except that the participants were told that the keypress depended on the stimulus' semantic category. The assignment of the animal and vegetable categories to the two responses was balanced across participants.

\section{Results and Discussion}

Mean RTs for correct responses and percentages of errors are presented in Table 2. RT data and errors were en- 
tered into ANOVAs with three within-participants factors: stimulus type (animal or vegetable), word length (four, five, six, or seven letters) and correspondence.

In the RT ANOVA, there was a significant effect of stimulus type $[F(1,41)=19.08, p<.0001]$. Responses to stimuli from the animal category were faster than those to stimuli from the vegetable category. Because frequency did not significantly differ between categories, some other variables (e.g., familiarity or imageability) must have rendered animal names easier to identify. Stimulus length was significant too $[F(3,123)=21.47, p<.0001]$ and interacted significantly with stimulus type $[F(3,123)=6.99$, $p<.0005]$. As in the previous experiment, there was also a significant effect of correspondence $[F(1,41)=8.61, p<$ $.01]$ : RTs were shorter on corresponding than on noncorresponding trials ( 777 vs. $792 \mathrm{msec}$; left- vs. right-response RTs for standard-oriented and mirrored stimuli were 699 vs. $706 \mathrm{msec}$ and $877 \mathrm{vs} .855 \mathrm{msec}$, respectively). A correspondence effect was found in the error ANOVA $[F(1,41)=$ $6.40, p<.05]$. Corresponding responses yielded a lower error rate than did noncorresponding responses $(4.7 \% \mathrm{vs}$. $6.0 \%$; left- vs. right-response error percentages for standard and mirrored stimuli were $2.7 \%$ vs. $4.3 \%$ and $5.6 \%$ vs. $4.8 \%$, respectively). Although this analysis did not reveal significant effects of stimulus length and type, these two factors significantly interacted $[F(3,123)=6.01, p<$ $.001]$. In the error ANOVA too, neither stimulus length nor type interacted with correspondence.

As in Experiment 1, both RTs and accuracy showed an effect of correspondence between initial letter position and response position, indicating that the processing of standardoriented and mirror-reversed words produces spatial codes, regardless of the nature of the stimuli and task.

\section{GENERAL DISCUSSION}

Both experiments showed a correspondence effect between position of the beginning part of standard-oriented

\section{Table 2}

Experiment 2 (Categorical Discrimination): Mean Reaction Times (RTs, in Milliseconds) and Percentages of Errors (PEs) As a Function of Stimulus Type, Stimulus Length, and Initial-Letter-Response Spatial Correspondence

\begin{tabular}{|c|c|c|c|c|}
\hline Type & $\begin{array}{c}\text { Length } \\
\text { (in Letters) }\end{array}$ & Correspondence & RT & $\mathrm{PE}$ \\
\hline \multirow[t]{8}{*}{ Animal name } & \multirow[t]{2}{*}{4} & Corresponding & 740 & 5.2 \\
\hline & & Noncorresponding & 754 & 6.2 \\
\hline & \multirow[t]{2}{*}{5} & Corresponding & 758 & 6.3 \\
\hline & & Noncorresponding & 807 & 9.1 \\
\hline & \multirow[t]{2}{*}{6} & Corresponding & 764 & 3.4 \\
\hline & & Noncorresponding & 777 & 3.8 \\
\hline & \multirow[t]{2}{*}{7} & Corresponding & 773 & 3.6 \\
\hline & & Noncorresponding & 793 & 3.6 \\
\hline \multirow[t]{8}{*}{ Vegetable name } & \multirow[t]{2}{*}{4} & Corresponding & 751 & 4.4 \\
\hline & & Noncorresponding & 764 & 5.8 \\
\hline & \multirow[t]{2}{*}{5} & Corresponding & 793 & 3.6 \\
\hline & & Noncorresponding & 784 & 6.2 \\
\hline & \multirow[t]{2}{*}{6} & Corresponding & 805 & 4.6 \\
\hline & & Noncorresponding & 806 & 7.9 \\
\hline & \multirow[t]{2}{*}{7} & Corresponding & 831 & 6.5 \\
\hline & & Noncorresponding & 849 & 5.6 \\
\hline
\end{tabular}

or mirror-reversed stimuli and response position. This finding suggests that the stimulus processing involved in the present experimental tasks led to spatial coding of the initial part of the stimulus, even though this was irrelevant to the tasks. The resulting spatial code, in turn, primed the corresponding response, thus causing interference at the response selection stage.

In contrast with the widely held hypothesis that reading words produces internally uniform representations, our results clearly indicate that these representations are biased toward the initial parts of words. The fact that the correspondence effect was found for both words and pronounceable nonwords suggests that the spatial coding of word beginnings occurs at a prelexical stage of word processing. Following Fischer (1996, 2000), this might result from the selective orienting of attention toward wordinitial letter(s) and might be related to lexical access. In this respect, the present results are compatible with the attention shift account of the Simon effect (e.g., Nicoletti \& Umiltà, 1994), according to which the spatial stimulus code that produces the Simon effect derives from the shift of attention toward the stimulus location. Alternatively, the word-beginning bias might be the consequence of differential weights being attributed to letters as a function of their position in the word, because of either perceptual mechanisms (Montant et al., 1998) or processes linked to letter position coding (Whitney, 2001).

Besides showing preferred coding of word-initial letters, the present results shed light on the nature of the coding. As has already been indicated, left-right asymmetries have been found in flanker tasks with standard-oriented and mirror-reversed letter strings: a greater effect of left and right flankers, respectively, which has been accounted for in terms of stimulus-scanning direction in reaching the central target (Hommel, 1995, 2003). Standard-oriented stimuli, for example, would induce a left-to-right scanning and, since the scanning process stops at the stimulus center, only left flankers would be processed. Hommel (2003) proposed that there might be some kind of mechanism that induces the cognitive system to start scanning a string from either the leftmost or the rightmost element, depending on the string orientation, that is activated when the string is classified as having a wordlike structure (i.e., it would be related to reading). One possibility is that this mechanism involves coding the position of what is perceived as the initial letter of the string; that is, the initial letter of wordlike stimuli is tagged in spatially organized stimulus representations.

However, evidence has never been provided that the mechanism proposed by Hommel (2003) occurs during reading. The present study provides the first demonstration that in word recognition, beginning letters have a special role and are coded in a spatial representation (see Caramazza \& Hillis, 1990) that reflects the actual orientation of the physical stimulus (i.e., they are on the left in the representation of standard-oriented words and on the right in those of mirror-reversed words). It follows that in reading, the direction of script is automatically processed and the word-beginning position is coded before the orthographic lexicon is accessed. 


\section{AUTHOR NOTE}

This study was supported by a grant from the University of Padua to B.T. and by Grant PRIN 2005 from MIUR to C.U. We thank Ken Forster, Stephen Goldinger, Bernhard Hommel, and Robert Proctor for their constructive comments on the manuscript. Correspondence concerning this article should be addressed to B. Treccani, Dipartimento di Psicologia Generale, Università di Padova, via Venezia 8, 35131 Padova, Italy (e-mail: barbara.treccani@unipd.it).

\section{REFERENCES}

Asso, D., \& WYKe, M. (1967). Experimental study of the effect of letter reversals on reading. British Journal of Psychology, 58, 413-419.

Brysbaert, M., Vitu, F., \& Schroyens, W. (1996). The right visual field advantage and the optimal viewing position effect: On the relation between foveal and parafoveal word recognition. Neuropsychology, 10, 385-395.

Burnage, G., \& Dunlop, D. (1993). Encoding the British National Corpus. In J. [M.] Aarts, P. de Haan, \& N. Oostdijk (Eds.), English language corpora: Design, analysis and exploitation (pp. 79-95). Amsterdam: Rodopi.

Caramazza, A., \& Hillis, A. E. (1990). Levels of representation, coordinate frames, and unilateral neglect. Cognitive Neuropsychology, 7, 391-445

Chokron, S., \& Imbert, M. (1993). Influence of reading habits on line bisection. Cognitive Brain Research, 1, 219-222.

Coltheart, M. (1981). The MRC Psycholinguistic Database. Quarterly Journal of Experimental Psychology, 33A, 497-505.

Cubelli, R., Lotto, L., Paolieri, D., Girelli, M., \& Job, R. (2005). Grammatical gender is selected in bare noun production: Evidence from the picture-word interference paradigm. Journal of Memory \& Language, 53, 42-59.

De Jong, R., Liang, C.-C., \& Lauber, E. (1994). Conditional and unconditional automaticity: A dual-process model of effects of spatial stimulus-response correspondence. Journal of Experimental Psychology: Human Perception \& Performance, 20, 731-750.

Ducrot, S., \& Pynte, J. (2002). What determines the eyes' landing position in words? Perception \& Psychophysics, 64, 1130-1144.

EriKsen, B. A., \& EriKsen, C. W. (1974). Effects of noise letters upon the identification of a target letter in a nonsearch task. Perception \& Psychophysics, 16, 143-149.

FARID, M., \& Grainger, J. (1996). How initial fixation position influences visual word recognition: A comparison of French and Arabic. Brain \& Language, 53, 351-368.

FisCHER, M. H. (1996). Bisection performance indicates spatial word representation. Cognitive Brain Research, 4, 163-170.

Fischer, M. H. (2000). Perceiving spatial attributes of print. In A. Kennedy, R. Radach, D. Heller, \& J. Pynte (Eds.), Reading as a perceptual process (pp. 89-117). Amsterdam: Elsevier.

Frederiksen, J. R., \& Kroll, J. F. (1976). Spelling and sound: Approaches to the internal lexicon. Journal of Experimental Psychology: Human Perception \& Performance, 2, 361-379.

Hommel, B. (1995). Attentional scanning in the selection of central targets from multi-symbol strings. Visual Cognition, 2, 119-144.

Hommel, B. (2003). Spatial asymmetries in the flanker-congruency effect: Attentional scanning is biased by flanker orientation. Psychological Science, 45, 63-77.
Inhoff, A. W., Pollatsek, A., Posner, M. I., \& Rayner, K. (1989). Covert attention and eye movements during reading. Quarterly Journal of Experimental Psychology, 41A, 63-89.

Johnson, R. L., Perea, M., \& Rayner, K. (2007). Transposed-letter effects in reading: Evidence from eye movements and parafoveal preview. Journal of Experimental Psychology: Human Perception \& Performance, 33, 209-229.

Koriat, A., \& Norman, J. (1985). Reading rotated words. Journal of Experimental Psychology: Human Perception \& Performance, 11, 490-508.

Kwantes, P. J., \& Mewhort, J. K. (1999). Evidence for sequential processing in visual word recognition. Journal of Experimental Psychology: Human Perception \& Performance, 25, 376-381.

LaBerge, D. (1983). Spatial extent of attention to letters and words. Journal of Experimental Psychology: Human Perception \& Performance, 9, 371-379.

McConkie, G. W., Kerr, P. W., Reddix, M. D., \& Zola, D. (1988). Eye movement control during reading: I. The location of initial eye fixations on words. Vision Research, 28, 1107-1118.

Montant, M., Nazir, T. A., \& Poncet, M. (1998). Pure alexia and the viewing position effect in printed words. Cognitive Neuropsychology, 15, 93-140

Nicoletti, R., \& Umiltà, C. (1994). Attention shifts produce spatial stimulus codes. Psychological Research, 56, 144-150.

Plaut, D. C., McClelland, J. L., Seidenberg, M. S., \& Patterson, K. (1996). Understanding normal and impaired word reading: Computational principles in quasi-regular domains. Psychological Review, 103, 56-115.

RAYNER, K. (1979). Eye guidance in reading: Fixation locations within words. Perception, 8, 21-30.

Schoonbaert, S., \& Grainger, J. (2004). Letter position coding in printed word perception: Effects of repeated and transposed letters. Language \& Cognitive Processes, 19, 333-367.

Sieroff, E., \& Posner, M. I. (1988). Cueing spatial attention during processing of words and letter strings in normals. Cognitive Neuropsychology, 5, 451-472.

Simon, J. R., \& Small, A. M., JR. (1969). Processing auditory information: Interference from an irrelevant cue. Journal of Applied Psychology, 53, 433-435.

Umiltà, C., \& Nicoletti, R. (1992). An integrated model of the Simon effect. In J. Alegria, D. Holender, J. Junça de Morais, \& M. Radeau (Eds.), Analytic approaches to human cognition (pp. 331-350). Amsterdam: Elsevier.

Vitu, F., O’Regan, J. K., \& Mittau, M. (1990). Optimal landing position in reading isolated words and continuous text. Perception \& Psychophysics, 47, 583-600.

VONK, W., RADACH, R., \& VAN RIJN, H. (2000). Eye guidance and the saliency of word beginnings in reading text. In A. Kennedy, R. Radach, D. Heller, \& J. Pynte (Eds.), Reading as a perceptual process (pp. 269-299). Amsterdam: Elsevier.

Whitney, C. (2001). How the brain encodes the order of letters in a printed word: The SERIOL model and selective literature review. Psychonomic Bulletin \& Review, 8, 221-243.

(Manuscript received February 2, 2007; revision accepted for publication July 9, 2007.) 